\title{
Effect of Charred Rice Husk on Rainfed Groundnut for Water Retention and Nutrient Management (Arachis hypogaea L.)
}

\author{
P. Balasubramanian* and C.R. Chinnamuthu
}

Department of Agronomy, AC\&RI, TNAU, Madurai, Tamil Nadu, India

\author{
*Corresponding author
}

\section{Keyw ords \\ Groundnut, Rainfed, Enriched charred rice husk, FYM, Lignite, SEM-EDAX and water retention capacity.}

\section{Article Info}

Accepted:

28 September 2017 Available Online: 10 November 2017

\section{A B S T R A C T}

A field experiment was conducted at the Department of Agronomy, Agricultural College and Research Institute, Tamil Nadu Agricultural University, Madurai, Tamil Nadu during 2014-2015 rabi season under rainfed situation to find out the effect of charred rice husk and other organic materials enriched with or without fertilizer nutrient on the moisture retention and nutrient supply during the deficit period of crop growth. The field experiment was conducted with ten treatments, replicated thrice laid out in randomized block design. Groundnut variety, VRI 2, a Spanish bunch type was selected for the study and raised under sandy clay loam textured soil. The treatment includes application of charred rice husk, biochar, lignite and farm yard manure alone and enriched with the recommended dose of nitrogen $\left(10 \mathrm{~kg} \mathrm{ha}^{-1}\right)$ and phosphorus (10 $\mathrm{kg} \mathrm{ha}^{-1}$ ) fertilizer and compared with the absolute control and recommended dose of fertilizer $\left(10: 10: 45 \mathrm{~kg} \mathrm{NPK} \mathrm{ha}{ }^{-1}\right)$. The structural morphology of organic materials were studied using Scanning Electron Microscope (SEM) showed that the particles of charred rice husk were uneven and the diameter varied from 3-4 $\mu \mathrm{m}$ with clod and block appearance. The particles of biochar were irregular in shape, uneven sized and diameter varied from 2-3 $\mu \mathrm{m}$ with block appearance and the lignite particles found spherical and aggregated. The chemical composition of intercalated manure with nutrient examined under SEM-EDAX revealed that the charred rice husk contains 64.31 per cent carbon 28.42 per cent oxygen and 5.96 per cent silica. Whereas the biochar composed of Carbon 66.65 per cent, nitrogen 6.58 per cent, oxygen 24.55 per cent and silica 0.26 per cent and lignite comprised of carbon (63.64 per cent), nitrogen ( 2.56 per cent), oxygen ( 24.96 per cent) and silica ( 1.25 per cent). The different organic manures at the rate of 5 tons/ ha ${ }^{-1}$ was mixed with the calculated quantities of urea and single super phosphate and incubated for 45 days. The nutrient loaded organic manures were incorporated into the dry soil during land preparation. Total amount of rainfall received during the entire crop growth period was $179 \mathrm{~mm}$ in 20142015. Data on crop growth characteristics revealed that, among various treatments; enriched farm yard manure recorded higher values when compared to other treatments. Application of enriched biochar and charred rice husk closely followed the best treatment with respect to biometric parameters at all stages of crop growth. Incorporation of enriched FYM at $5 \mathrm{t} \mathrm{ha}^{-1}$ recorded significantly higher pod yield $\left(2190 \mathrm{~kg} \mathrm{ha}^{-1}\right)$. The enriched biochar and charred rice husk produced comparable pod yield of $2010 \mathrm{~kg} \mathrm{ha}^{-1}$ and $1983 \mathrm{~kg} \mathrm{ha}^{-1}$, respectively. 


\section{Introduction}

Groundnut (Arachis hypogaea L.), the 'King of oilseeds' is an unpredictable legume, may continue to be an important commercial crop in rainfed areas. About 69 per cent of the groundnut area is under rainfed generating 53.42 per cent of the total production (Directorate of Economics and Statistics, Department of Agriculture and Cooperation, 2009-10). The uncertainty of groundnut productivity in the rainfed areas could be minimized by in situ conserving the soil moisture received through precipitation during the cropping period and improving the nutrient status.

Rice husk is the natural sheath or productive cover, which forms the cover of rice grains during their growth. Rice husk represents about 20 per cent by the weight of the rice harvested. About 80 per cent by weight of the raw husk is made of organic components (Anonymous, 1979) and incorporation of rice husk into soil mixture was found to affect many crops (Sharma et al., 1988).

Now days, Soil organic matter content is gradually declining due to high cropping intensity which causes quick decomposition of organic matter. Use of rice husk as an organic manure, might be play a vital role not only in improving soil physical condition but also in improving the plant nutrients. Incorporation of rice husk can significantly improve soil properties by decreasing soil bulk density, enhancing soil $\mathrm{pH}$, adding organic carbon, increasing available nutrients and removing heavy metals from the system, ultimately increasing crop yields (Williams et al., 1972). Rice husk under different irrigation intervals can give good rice stand, better grain yield and higher water use efficiency (AboSoliman et al., 1990). The present investigation was aimed to study the role of enriched rice husk as an organic manure on groundnut pod yield and water retention capacity under rainfed condition.

\section{Materials and Methods}

Field experiment was conducted during rabi 2014-2015 under rainfed condition at the Central Farm, Department of Agronomy, Agriculture College and Research Institute, Madurai, Tamil Nadu. The site was located at $9^{0}$. 54' $\mathrm{N}$ latitude and $78^{\circ} .80^{\prime} \mathrm{E}$ longitude at an altitude of $147 \mathrm{~m}$ above mean sea level. The region falls under the southern zone of Tamil Nadu. Field experiment was conducted with ten treatments (Figure.2) replicated thrice laid out in randomized block design. The treatment includes application of charred rice husk, biochar, lignite and farm yard manure alone and enriched with the recommended dose of nitrogen $\left(10 \mathrm{~kg} \mathrm{ha}^{-1}\right)$ and phosphorus $\left(10 \mathrm{~kg} \mathrm{ha}^{-1}\right)$ fertilizer and compared with the absolute control and recommended dose of fertilizer $(10: 10: 45 \mathrm{~kg}$ NPK $\left.\mathrm{ha}^{-1}\right)$. The enriched organic manures were prepared by taking each at the rate of 5 tons/ $\mathrm{ha}^{-1}$ and mixed thoroughly with the recommended dose of urea and phosphorus. The mixture was incubated for 45 days in dark room. The procedure for loading nutrient in the organic material is detailed below (Figure.1).

Groundnut variety, VRI 2, was selected for the study and sown at $30 \times 10 \mathrm{~cm}$ spacing to maintain the uniform plant population of 40 plants per $\mathrm{m}^{2}$ area. Seeds were uniformly coated with Trichoderma viride at the rate of $4 \mathrm{~g} \mathrm{~kg}^{-1}$ and dried in shade before sowing. Soil samples, from the experimental sites, as well as enriched organic manures were collected and analyzed for chemical composition. The results, for both soil and enriched organic manures, were presented in Table (1). Total amount of rainfall received during the entire crop growth period (November 2014 to January 2015) was 179 mm. 


\section{Scanning electron microscope (SEM) study}

The scanning electron microscope (SEM) is a type of electron microscope that images the samples surface by scanning it with a highenergy beam of electrons in a raster scan pattern. Here, a wide range of magnifications is possible, from 10 times (equivalent to that of a powerful hand-lens) to more than 5, 00,000 times. All samples must be of an appropriate size to fit in the specimen chamber and are generally mounted rigidly on a specimen holder called a specimen stub.

For taking images of sample, about 0.5 to 1.0 $\mathrm{mg}$ of sample was dusted on the carbon conducting tape. Then the tape was mounted on sample stage and the images were taken in 24,000X magnification and 15 to $30 \mathrm{KV}$ using FEI ESEM Model "QUANTA 250" available in the Department of Nano Science and Technology, Tamil Nadu Agricultural University, Coimbatore. Energy Dispersive $\mathrm{X}$-Ray Spectroscopy (EDAX) is a chemical micro analysis technique used in conjunction with scanning electron microscopy (SEM). For recording the chemical composition of sample, about 0.5 to $1.0 \mathrm{mg}$ of sample was dusted on the carbon conducting tape.

\section{Water releasing pattern}

Water release pattern of different organic manures were estimated by leaching columns were constructed from commercially available PVC plastic pipe $(75 \mathrm{~mm})$ with suitable length of gap into the base assembly. White colour nylon cloth was placed on the base to prevent loss of fine materials. The leaching water were pored to the columns from plastic dropping funnels and collected from the base of the columns in $500 \mathrm{ml}$ conical flask (James et al., 2007).Other cultural practices was followed as recommended by Tamil Nadu Agricultural University, Tamil Nadu.
Agronomic biometric observation and analysis study

Five plants were randomly selected from each treatments to measure the agronomic parameters. All data collected for experimental purposes were statistically analyzed by analysis of variance (ANOVA) using AGRES (Data Entry Module for AgRes Statistical software version 3.01, 1994 Pascal Intl. Software Solutions). Differences between mean values were evaluated for significance using Least Significant Difference (LSD) at 5 per cent probability level as suggested by Gomez and Gomez (1984).

\section{Results and Discussion}

The structural morphology of charred rice husk, biochar and lignite were studied using Scanning Electron Microscope (SEM) at low and high resolutions. The porous rough surface characteristic of organic materials aided in loading of nitrogen and phosphorus due to the physical adsorption and also by covalent bonding with energy dispersive $\mathrm{X}$ ray spectroscopy (EDAX) (Fig. 3).The chemical composition of intercalated manure with nutrient was examined under SEMEDAX. The EDAX data confirmed the composition of charred rice husk, biochar and lignite.

\section{Growth characters}

Concerning, application of organic manures, results in Table (2) clearly showed that a significant positive effect was observed on all traits at rates of 5 tons $\mathrm{ha}^{-1}$ which increased vegetative growth traits. The results in Table (2) showed that plant height $(\mathrm{cm})$ and number of branches plant ${ }^{-1}$ and leaf area index were significantly affected by the absolute control plot. The highest values were recorded under enriched farm yard manure 5 tons $\mathrm{ha}^{-1}$ 
followed by enriched charred rice husk 5 tons $\mathrm{ha}^{-1}$. However, no significant difference was found between the enriched bio char and enriched lignite at 5 tons $\mathrm{ha}^{-1}$ on plant height (cm) and number of branches/plant on this traits. The same trend was observed on leaf area index. Aliyu et al., (2011) reported that application of rice husk as a manures two weeks before planting also produced the highest number of leaves in cowpea Chandrasekaran et al., (2007).

However, absolute control plots significantly reduced the values of all the studied growth attributes. This may be due to the decreasing moisture content in root zone for a long period, which adversely affected cell division, elongation and vegetative growth. The increase in branch number was attributed to the gradual release of nutrients during decomposition of manures (Budhar and Palaniappan, 1994 and Budhar, 2003).

\section{Pod yield and yield components}

With respect of yield and yield components such as, flowering percentage, number of pegs, number of pods, number of matured pods, single seeded pods and double seeded pods and hundred pod weight were different organic manures application plots Table (3). The results indicated that highly significant reduction was found in all the yield components with respect to without organic manures (absolute control plot), compared to the different enriched organic manures treatments Table (3). The results indicated that, application of enriched farm yard manure and enriched charred rice husk 5 tons $\mathrm{ha}^{-1}$ yielded the highest biological and pod yields respectively. Consequently, the increase in pod yield components can be due to the fact that available more water enhanced nutrient availability which improved nitrogen and other macro and micro elements absorption as well as enhancing the production and translocation of the dry matter content from source to sink. Similar results were reported by El Wehishy and Abd El Hafez (1997), respectively. Okon et al., (2005) stated that the optimum level of rice husk plus 0.05 ton urea ha- 1 can sustain rapid growth and better yield of okra even faster than NPK, because rice husk ash contains almost all other essential plant nutrients and the presence of nitrogen will boost their uptake. The increase in both biological and yields indicates that, rice husk already decomposed and its nitrogen as well as other nutrients was released to the plant, furthermore, improved physical and chemical soil properties could enhance the absorption of native nutrients in the soil. Similar results were found by Ebaid et al., (2005). The increase in biological yield could be due to the increase in yield attributes (plant height, number of branches, leaf area index and hundred pod weight) were stated Awad (2001) and El Refaee et al., (2006). However, no significant difference were found between enriched bio char and enriched lignite at 5 tons $\mathrm{ha}^{-1}$.

On the other hand, without organic manures recorded the lowest biological and pod yields. These results revealed that the reduction in yield components can be expected as plants are exposed to water deficit. Besides, available water enhanced the production and transporting of dry matter content to the pod yield resulting in more pod yield. This is in agreement with results reported by Nour et al., (1996).

\section{Nutrient uptake by crops}

Increased trend in nutrient uptake of NPK from 30, 60, 90 DAS and harvest stage of crops Table (5) were found higher due to the incorporation of enriched FYM at 5 tons $\mathrm{ha}^{-1}$ which was followed by enriched biochar and charred rice husk at 5 tons ha ${ }^{-1}$. This could be 
due to the role of different organic manures as organic fertilizer on better holding the water in the root zone. The reason might be due to the increased root length and root volume which might have tapped the available nutrients from rhizosphere at the increased soil moisture level than non-application of organic manure plots. The other reason could be that the applied organics might have created favorable physical, chemical and microbial environment. Furthermore, improved physical and chemical soil properties could enhance the absorption of native nutrients in the soil. (Ebaid et al., 2005). Gupta et al., (1988) reported that available $\mathrm{N}$ content of the soil increased significantly with increasing application of FYM.

Fig.1 Procedure for enriched organic manures preparation

Charred rice husk loaded with urea at the recommended dose and mixed thoroughly

Further the single super phosphate at the recommended dose mixed with Charred rice husk

Covered with Tar paulin and incubated for 45 days at room temperature

Resulted in enriched manure with nitrogen and phosphorus

Fig.2 Treatments Details

$\mathbf{T}_{1} \quad$ - Charred rice husk $+100 \%$ recommended dose of $\mathrm{N}$ and $\mathrm{P}$ through soil application

$\mathbf{T}_{2}-$ Biochar $+100 \%$ recommended dose of $\mathrm{N}$ and $\mathrm{P}$ through soil application

$\mathbf{T}_{3} \quad$ - Lignite $+100 \%$ recommended dose of $\mathrm{N}$ and $\mathrm{P}$ through soil application

$\mathbf{T}_{4} \quad$ Farm yard manure $+100 \%$ recommended dose of $\mathrm{N}$ and $\mathrm{P}$ through soil application

$\mathbf{T}_{5} \quad$ - Charred rice husk 5 tons $\mathrm{ha}^{-1}$

$\mathbf{T}_{6} \quad$ - Biochar 5 tons ha ${ }^{-1}$

$\mathbf{T}_{7} \quad$ - Farm yard manure 5 tons ha $^{-1}$

$\mathbf{T}_{\mathbf{8}} \quad$ - Lignite 5 tons $\mathrm{ha}^{-1}$

$\mathbf{T}_{9} \quad-$ Absolute Control

$\mathbf{T}_{\mathbf{1 0}} \quad$ - Recommended dose of fertilizer (RDF) (10:10:45 $\left.\mathrm{kg} \mathrm{NPK} \mathrm{ha}^{-1}\right)$

Note: Urea contains $46 \% \mathrm{~N}$, Single super phosphate contains $16 \% \mathrm{P}_{2} \mathrm{O}_{4}$ and $12 \% \mathrm{SO}_{4}$ and Muriate of potash contains $60 \% \mathrm{~K}_{2} \mathrm{O}$. 
Fig.3 Scanning Electron Microscope (SEM) of the size reduced charred rice husk a) $30 \mu \mathrm{m}$ b) $10 \mu \mathrm{m}$ resolution (SEM)
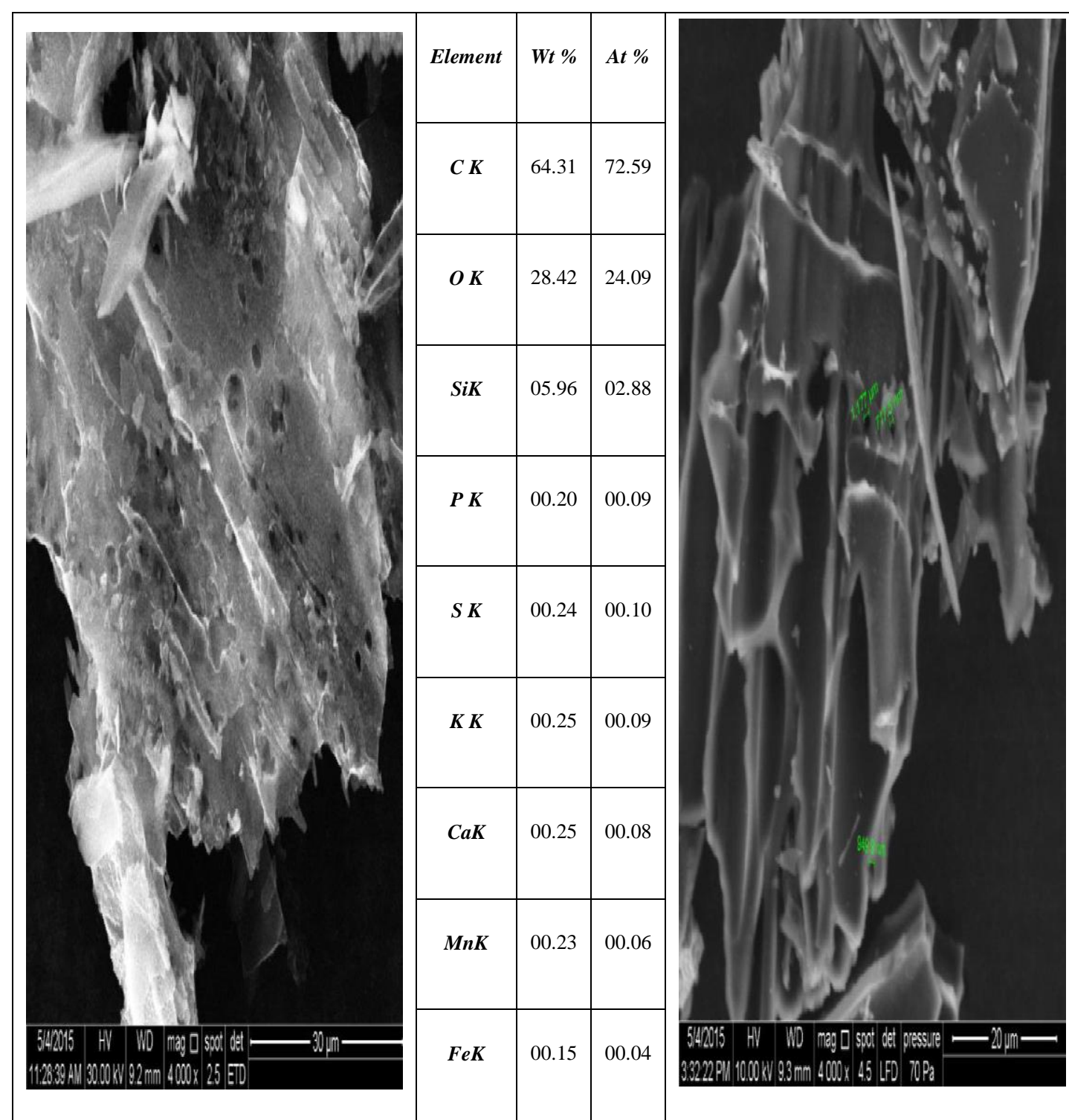

\begin{tabular}{|c|c|c|}
\hline Element & $W t \%$ & At \% \\
\hline$C K$ & 66.65 & 72.87 \\
\hline$N K$ & 03.58 & 06.17 \\
\hline$O K$ & 24.55 & 20.15 \\
\hline $\mathrm{NaK}$ & 00.08 & 00.04 \\
\hline $\mathrm{SiK}$ & 00.26 & 00.12 \\
\hline$P K$ & 00.23 & 00.10 \\
\hline$S K$ & 00.30 & 00.12 \\
\hline$K K$ & 00.43 & 00.14 \\
\hline CaK & 00.63 & 00.21 \\
\hline
\end{tabular}

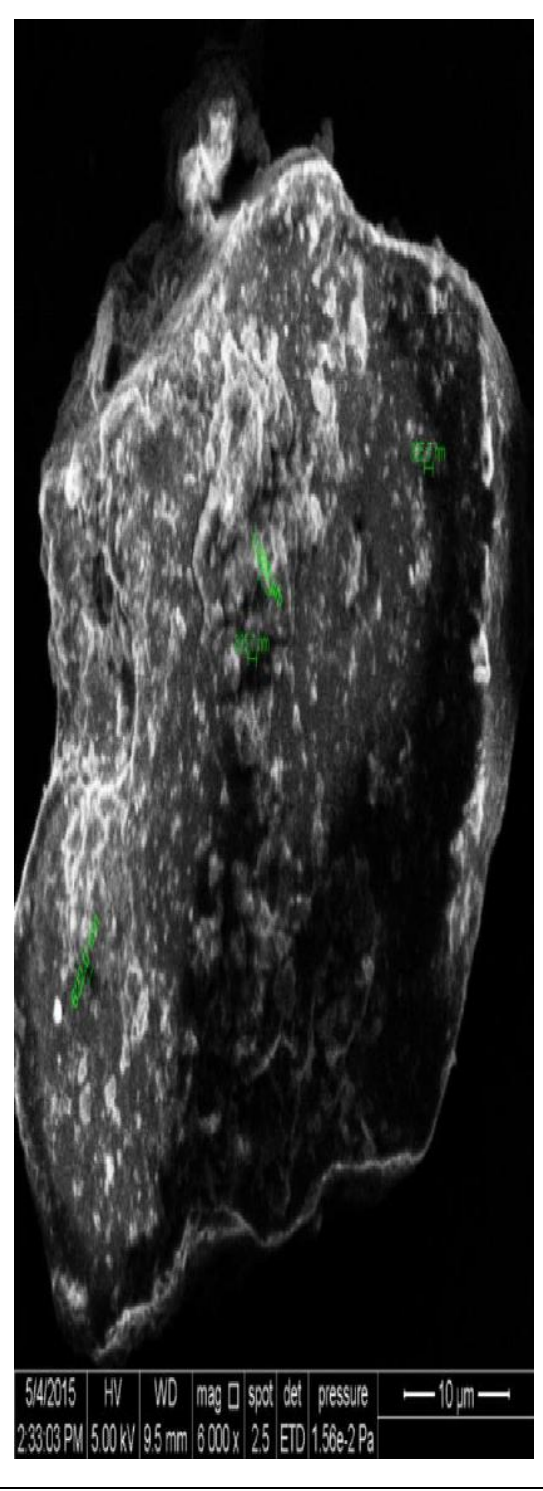

\begin{tabular}{|c|c|c|}
\hline Element & $W t \%$ & At \% \\
\hline$C K$ & 63.64 & 72.07 \\
\hline$N K$ & 01.56 & 02.49 \\
\hline$O K$ & 24.96 & 21.22 \\
\hline $\mathrm{NaK}$ & 00.49 & 00.29 \\
\hline$A l K$ & 05.32 & 02.68 \\
\hline $\mathrm{SiK}$ & 01.05 & 00.51 \\
\hline$P K$ & 00.22 & 00.09 \\
\hline$S K$ & 00.61 & 00.26 \\
\hline$K K$ & 00.36 & 00.13 \\
\hline
\end{tabular}


Table.1 Soil properties of the experimental sites before sowing and chemical composition of charred rice husk sample

\begin{tabular}{|c|c|c|c|c|c|}
\hline \multicolumn{2}{|l|}{ Soil sample } & \multirow{2}{*}{\multicolumn{3}{|c|}{$\begin{array}{c}\text { Charred rice husk } \\
\text { Nutrient content (Per cent) }\end{array}$}} & Lignite \\
\hline Properties & $2014-2015$ & & & & \\
\hline Clay (Per cent) & 21.45 & Carbon & 64.31 & 66.65 & 63.64 \\
\hline Silt (Per cent) & 9.05 & Oxygen & 28.42 & 24.55 & 24.96 \\
\hline Bulk density $\left(\mathrm{g} \mathrm{cm}^{3}\right)$ & 1.38 & Nitrogen & 0.2 & 3.58 & 1.56 \\
\hline Particle density $\left(\mathrm{g} \mathrm{cm}^{3}\right)$ & 2.08 & Phosphorus & 0.20 & 0.23 & 0.22 \\
\hline Available soil moisture $(\mathrm{mm} / 60 \mathrm{~cm})$ & 101.05 & Potassium & 0.25 & 0.43 & 0.36 \\
\hline Field capacity (Per cent) & 20.07 & Calcium & 0.25 & 0.63 & 0.78 \\
\hline Available $\mathrm{N} \mathrm{kg} \mathrm{ha}^{-1}$ (Subbiah and Asija, 1956) & 154.00 & Sulphur & 0.24 & 0.30 & 0.61 \\
\hline Available $\mathrm{P} \mathrm{kg} \mathrm{ha}^{-1}$ (Olsen et al., 1954) & 20.00 & Manganese & 0.23 & 0.00 & 0.00 \\
\hline Available $\mathrm{K} \mathrm{kg} \mathrm{ha}^{-1}$ (Stanford and English, 1949) & 195.00 & Iron & 0.15 & 0.00 & 0.00 \\
\hline Organic carbon (per cent) (Walkley and Black, 1934) & 0.44 & zinc & 0.00 & 0.00 & 0.00 \\
\hline pH (1:2 soil water suspension) (Jackson, 1973) & 7.50 & Copper & 0.00 & 0.00 & 0.00 \\
\hline $\begin{array}{l}\text { Electrical conductivity }\left(\mathrm{dSm}^{-1}\right)(1: 2 \text { soil water } \\
\text { suspension) (Jackson, } 1973)\end{array}$ & 0.42 & Silica & 5.96 & 0.26 & 1.05 \\
\hline
\end{tabular}

Table. 2 Effect of enriched charred rice husk and other organic materials on Growth characters

\begin{tabular}{|c|c|c|c|}
\hline Treatments & Plant height $(\mathrm{cm})$ & No. of branches/ plant $^{-1}$ & Leaf Area Index \\
\hline $\mathrm{T}_{1}(\mathrm{CRH}+\mathrm{NP})$ & 48.59 & 11.84 & 4.60 \\
\hline $\mathrm{T}_{2}(\mathrm{BC}+\mathrm{NP})$ & 46.62 & 11.94 & 4.38 \\
\hline $\mathrm{T}_{3}(\mathrm{LN}+\mathrm{NP})$ & 43.16 & 11.07 & 4.16 \\
\hline $\mathrm{T}_{4}(\mathrm{FYM}+\mathrm{NP})$ & 52.49 & 13.15 & 4.89 \\
\hline $\mathrm{T}_{5}(\mathrm{CRH})$ & 41.97 & 10.16 & 3.43 \\
\hline $\mathrm{T}_{6}(\mathrm{BC})$ & 41.56 & 10.27 & 3.55 \\
\hline $\mathrm{T}_{7}(\mathrm{FYM})$ & 42.98 & 10.53 & 4.01 \\
\hline $\mathrm{T}_{8}(\mathrm{LN})$ & 39.00 & 10.04 & 3.23 \\
\hline $\mathrm{T}_{9}$ (Control) & 36.04 & 7.92 & 2.87 \\
\hline $\mathrm{T}_{10}(\mathrm{RDF} N \mathrm{NP})$ & 44.34 & 11.24 & 4.66 \\
\hline SEd & 1.08 & 0.18 & - \\
\hline CD (p=0.05) & 2.27 & 0.39 & - \\
\hline
\end{tabular}

Table.3 Effect of enriched charred rice husk and other organic materials on yield characters

\begin{tabular}{|c|c|c|c|c|c|c|c|}
\hline Treatments & $\begin{array}{c}\text { Flowering } \\
\text { percentage } \\
\text { plant }^{-1}\end{array}$ & $\begin{array}{c}\text { Number } \\
\text { of pegs } \\
\text { plant }^{-1}\end{array}$ & $\begin{array}{c}\text { Number } \\
\text { of pods } \\
\text { plant }^{-1}\end{array}$ & $\begin{array}{c}\text { Number of } \\
\text { matured pods } \\
\text { plant }^{-1}\end{array}$ & $\begin{array}{c}\text { Single } \\
\text { seeded pods } \\
\text { plant }^{-1}\end{array}$ & $\begin{array}{c}\text { Double } \\
\text { seeded pods } \\
\text { plant }^{-1}\end{array}$ & $\begin{array}{c}\text { Hundred } \\
\text { pod weight } \\
\text { (g) }\end{array}$ \\
\hline $\mathrm{T}_{1}(\mathrm{CRH}+\mathrm{NP})$ & 8.50 & 28.81 & 23.19 & 19.78 & 3.10 & 16.79 & 101.27 \\
\hline $\mathrm{T}_{2}(\mathrm{BC}+\mathrm{NP})$ & 8.47 & 29.47 & 23.26 & 20.14 & 2.99 & 17.45 & 101.54 \\
\hline $\mathrm{T}_{3}(\mathrm{LN}+\mathrm{NP})$ & 7.84 & 26.67 & 20.08 & 17.09 & 2.13 & 14.51 & 100.03 \\
\hline $\mathrm{T}_{4}(\mathrm{FYM}+\mathrm{NP})$ & 9.16 & 31.78 & 25.77 & 22.97 & 3.11 & 20.22 & 102.95 \\
\hline $\mathrm{T}_{5}(\mathrm{CRH})$ & 6.20 & 22.87 & 18.03 & 14.02 & 4.20 & 10.54 & 99.78 \\
\hline $\mathrm{T}_{6}(\mathrm{BC})$ & 6.88 & 23.42 & 19.21 & 14.86 & 3.14 & 11.46 & 99.81 \\
\hline $\mathrm{T}_{7}(\mathrm{FYM})$ & 7.24 & 24.16 & 20.48 & 16.24 & 4.01 & 11.78 & 100.01 \\
\hline $\mathrm{T}_{8}(\mathrm{LN})$ & 6.16 & 21.84 & 16.94 & 12.40 & 4.00 & 8.27 & 98.83 \\
\hline $\mathrm{T}_{9}($ Control $)$ & 5.16 & 15.70 & 11.41 & 9.56 & 4.65 & 5.84 & 97.23 \\
\hline $\mathrm{T}_{10}(\mathrm{RDF}$ NPK $)$ & 7.60 & 27.48 & 21.48 & 16.21 & 3.57 & 13.72 & 100.48 \\
\hline SEd & 0.17 & 0.41 & 0.47 & 2.40 & 0.07 & 0.22 & NS \\
\hline $\mathrm{CD}(\mathrm{p}=0.05)$ & 0.36 & 0.87 & 0.99 & 5.05 & 0.15 & 0.47 & \\
\hline
\end{tabular}


Table.4 Biological and pod yields as well as harvest index as affected by enriched charred rice husk and other organic materials

\begin{tabular}{|c|c|c|c|}
\hline Treatments & Pod yield kg/ ha ${ }^{-1}$ ) & Haulm yield $\left(\mathrm{kg} \mathrm{ha}^{-1}\right)$ & Harvest Index \\
\hline $\mathrm{T}_{1}(\mathrm{CRH}+\mathrm{NP})$ & 2010 & 5115 & 62.81 \\
\hline $\mathrm{T}_{2}(\mathrm{BC}+\mathrm{NP})$ & 1983 & 5118 & 62.17 \\
\hline $\mathrm{T}_{3}(\mathrm{LN}+\mathrm{NP})$ & 1800 & 4897 & 60.30 \\
\hline $\mathrm{T}_{4}(\mathrm{FYM}+\mathrm{NP})$ & 2190 & 5304 & 63.62 \\
\hline $\mathrm{T}_{5}(\mathrm{CRH})$ & 1500 & 4389 & 57.84 \\
\hline $\mathrm{T}_{6}(\mathrm{BC})$ & 1559 & 4399 & 57.17 \\
\hline $\mathrm{T}_{7}(\mathrm{FYM})$ & 1681 & 4628 & 58.64 \\
\hline $\mathrm{T}_{8}(\mathrm{LN})$ & 1486 & 4125 & 55.01 \\
\hline $\mathrm{T}_{9}$ (Control) & 1250 & 3849 & 51.46 \\
\hline $\mathrm{T}_{10}(\mathrm{RDF}$ NPK) & 1900 & 5018 & 62.30 \\
\hline SEd & 84.58 & 112.64 & - \\
\hline $\mathrm{CD}(\mathrm{p}=0.05)$ & 177.70 & 236.66 & - \\
\hline
\end{tabular}

Table.5 Effect of enriched charred rice husk and other organic materials on nutrient uptake by plants

\begin{tabular}{|c|c|c|c|c|c|c|c|c|c|c|c|c|}
\hline & \multicolumn{4}{|c|}{ Available Nitrogen } & \multicolumn{4}{c|}{ Available Phosphorus } & \multicolumn{4}{|c|}{ Available Potassium } \\
\hline Treatments & 30 DAS & 60 DAS & 90 DAS & Harvest & 30 DAS & 60 DAS & 90 DAS & Harvest & 30 DAS & 60 DAS & 90 DAS & Harvest \\
\hline $\mathrm{T}_{1}($ CRH+NP) & 27.89 & 58.96 & 129.52 & 125.43 & 11.26 & 16.48 & 33.42 & 30.81 & 23.41 & 38.49 & 77.86 & 85.24 \\
\hline $\mathrm{T}_{2}(\mathrm{BC}+\mathrm{NP})$ & 28.22 & 59.27 & 130.95 & 126.89 & 11.89 & 17.27 & 33.94 & 31.23 & 23.86 & 39.12 & 78.52 & 86.13 \\
\hline $\mathrm{T}_{3}(\mathrm{LN}+\mathrm{NP})$ & 26.18 & 55.21 & 126.28 & 122.21 & 9.57 & 13.54 & 29.59 & 28.61 & 21.84 & 35.05 & 74.16 & 82.49 \\
\hline $\mathrm{T}_{4}(\mathrm{FYM}+\mathrm{NP})$ & 30.15 & 62.73 & 134.57 & 129.82 & 12.63 & 20.02 & 35.85 & 33.83 & 25.48 & 42.86 & 81.88 & 89.78 \\
\hline $\mathrm{T}_{5}(\mathrm{CRH})$ & 24.28 & 46.78 & 118.41 & 115.21 & 8.29 & 11.85 & 22.41 & 20.41 & 15.47 & 29.04 & 62.48 & 74.59 \\
\hline $\mathrm{T}_{6}(\mathrm{BC})$ & 24.31 & 47.19 & 119.59 & 116.42 & 8.88 & 12.26 & 23.12 & 21.83 & 16.28 & 29.47 & 63.12 & 75.67 \\
\hline $\mathrm{T}_{7}(\mathrm{FYM})$ & 25.93 & 50.24 & 122.65 & 118.69 & 10.21 & 13.52 & 25.68 & 24.16 & 18.46 & 32.49 & 66.98 & 78.69 \\
\hline $\mathrm{T}_{8}(\mathrm{LN})$ & 22.46 & 42.71 & 116.21 & 112.37 & 7.18 & 10.29 & 20.07 & 17.21 & 12.49 & 26.69 & 59.10 & 71.37 \\
\hline $\mathrm{T}_{9}$ (Control) & 20.11 & 38.38 & 110.93 & 106.33 & 6.81 & 8.92 & 16.49 & 14.55 & 11.46 & 23.20 & 56.34 & 67.55 \\
\hline $\mathrm{T}_{10}(\mathrm{RDF}-\mathrm{NPK})$ & 27.46 & 57.49 & 127.49 & 122.57 & 11.28 & 16.88 & 32.78 & 27.69 & 22.48 & 38.17 & 75.28 & 83.29 \\
\hline SEd & $\mathbf{0 . 5 9}$ & $\mathbf{0 . 9 3}$ & $\mathbf{2 . 9 6}$ & $\mathbf{2 . 5 1}$ & $\mathbf{0 . 1 9}$ & $\mathbf{0 . 3 5}$ & $\mathbf{0 . 6 2}$ & $\mathbf{0 . 4 0}$ & $\mathbf{0 . 2 4}$ & $\mathbf{0 . 7 8}$ & $\mathbf{1 . 1 8}$ & $\mathbf{1 . 5 6}$ \\
\hline CD $(\mathbf{p}=\mathbf{0 . 0 5})$ & $\mathbf{1 . 2 4}$ & $\mathbf{1 . 9 6}$ & $\mathbf{6 . 2 2}$ & $\mathbf{5 . 2 7}$ & $\mathbf{0 . 4 0}$ & $\mathbf{0 . 7 4}$ & $\mathbf{1 . 3 1}$ & $\mathbf{0 . 8 4}$ & $\mathbf{0 . 5 1}$ & $\mathbf{1 . 6 4}$ & $\mathbf{2 . 4 9}$ & $\mathbf{3 . 2 9}$ \\
\hline
\end{tabular}

Table.6 Effect of enriched charred rice husk and other organic materials on available soil moisture, Relative water content and Root nodules of rainfed groundnut

\begin{tabular}{|c|c|c|c|c|c|c|c|c|}
\hline & \multicolumn{4}{|c|}{ Available soil moisture (\%) } & \multicolumn{3}{c|}{ Relative water content } & \multicolumn{2}{c|}{ Root nodules } \\
\hline Treatments & 30 DAS & $\mathbf{6 0}$ DAS & $\mathbf{9 0}$ DAS & Harvest stage & 30 DAS & 60 DAS & 90 DAS & Harvest stage \\
\hline $\mathrm{T}_{1}(\mathrm{CRH}+\mathrm{NP})$ & 31.77 & 33.18 & 26.60 & 30.18 & 80.90 & 86.21 & 84.21 & 147.73 \\
\hline $\mathrm{T}_{2}(\mathrm{BC}+\mathrm{NP})$ & 29.71 & 31.56 & 24.46 & 27.89 & 78.93 & 84.00 & 82.47 & 142.80 \\
\hline $\mathrm{T}_{3}(\mathrm{LN}+\mathrm{NP})$ & 26.48 & 27.41 & 21.19 & 25.48 & 78.76 & 82.14 & 79.25 & 134.40 \\
\hline $\mathrm{T}_{4}(\mathrm{FYM}+\mathrm{NP})$ & 25.96 & 25.48 & 19.78 & 23.14 & 76.72 & 79.65 & 73.46 & 150.87 \\
\hline $\mathrm{T}_{5}(\mathrm{CRH})$ & 27.89 & 30.94 & 25.68 & 27.38 & 78.29 & 82.27 & 76.15 & 120.64 \\
\hline $\mathrm{T}_{6}(\mathrm{BC})$ & 26.15 & 28.47 & 22.56 & 26.47 & 78.54 & 78.65 & 75.27 & 119.73 \\
\hline $\mathrm{T}_{7}(\mathrm{FYM})$ & 24.95 & 24.69 & 19.76 & 23.24 & 76.72 & 75.14 & 70.16 & 131.57 \\
\hline $\mathrm{T}_{8}(\mathrm{LN})$ & 23.16 & 25.51 & 17.64 & 20.55 & 77.14 & 73.89 & 69.87 & 106.20 \\
\hline $\mathrm{T}_{9}(\mathrm{Control})$ & 21.48 & 21.18 & 14.79 & 16.49 & 62.67 & 69.54 & 65.42 & \\
\hline $\mathrm{T}_{10}(\mathrm{RDF}-\mathrm{NPK})$ & 22.49 & 23.74 & 17.33 & 18.72 & 75.49 & 72.14 & 67.46 & 100.42 \\
\hline SEd & $\mathbf{0 . 6 4}$ & $\mathbf{0 . 6 3}$ & $\mathbf{0 . 3 4}$ & $\mathbf{0 . 5 1}$ & $\mathbf{1 . 7 6}$ & $\mathbf{1 . 4 3}$ & $\mathbf{1 . 4 2}$ & $\mathbf{2 . 5 7}$ \\
\hline CD $(\mathbf{p}=\mathbf{0 . 0 5})$ & $\mathbf{1 . 3 6}$ & $\mathbf{1 . 3 2}$ & $\mathbf{0 . 7 1}$ & $\mathbf{1 . 0 7}$ & $\mathbf{3 . 6 9}$ & $\mathbf{3 . 0 1}$ & $\mathbf{2 . 9 8}$ & \\
\hline
\end{tabular}


Table.7 Effect of enriched charred rice husk and other organic materials on water uptake of rainfed groundnut $\mathrm{ml} / 500 \mathrm{ml}$

\begin{tabular}{|l|l|l|l|l|l|l|}
\hline \multirow{2}{*}{ Treatments } & \multicolumn{3}{|c|}{ Soil + organic manure 1.67(g) } & \multicolumn{2}{c|}{ Soil + 100\% organic manure 167(g) } \\
\cline { 2 - 7 } & $\begin{array}{c}\text { First day } \\
(\mathbf{m l})\end{array}$ & $\begin{array}{c}\text { Second day } \\
(\mathbf{m l})\end{array}$ & $\begin{array}{c}\text { Third day } \\
(\mathbf{m l})\end{array}$ & $\begin{array}{c}\text { First day } \\
(\mathbf{m l})\end{array}$ & $\begin{array}{c}\text { Second day } \\
(\mathbf{m l})\end{array}$ & $\begin{array}{c}\text { Third day } \\
(\mathbf{m l})\end{array}$ \\
\hline $\mathrm{T}_{1}(\mathrm{CRH}+\mathrm{NP})$ & 179.33 & 397.33 & 418.67 & 55.67 & 508.00 & 419.33 \\
\hline $\mathrm{T}_{2}(\mathrm{BC}+\mathrm{NP})$ & 185.10 & 425.33 & 442.00 & 128.67 & 331.33 & 442.67 \\
\hline $\mathrm{T}_{3}(\mathrm{LN}+\mathrm{NP})$ & 207.50 & 458.33 & 470.27 & 172.67 & 321.67 & 459.00 \\
\hline $\mathrm{T}_{4}(\mathrm{FYM}+\mathrm{NP})$ & 222.62 & 478.00 & 472.00 & 262.67 & 381.33 & 471.33 \\
\hline $\mathrm{T}_{9}(\mathrm{Control})$ & 270.67 & 485.00 & 478.00 & - & - & - \\
\hline $\mathrm{SEd}$ & 2.92 & 40.10 & 9.22 & 3.41 & 2.64 & 7.99 \\
\hline $\mathrm{CD}(\mathrm{p}=0.05)$ & 5.99 & 82.45 & 18.97 & 7.02 & 5.43 & 16.43 \\
\hline
\end{tabular}

Table.8 Effect of enriched charred rice husk and other organic materials on economics of cultivation of rainfed groundnut

\begin{tabular}{|l|c|c|c|c|}
\hline \multicolumn{1}{|c|}{ Treatments } & $\begin{array}{c}\text { Costs of cultivation } \\
\left(\text { Rs. ha }^{-\mathbf{1}}\right)\end{array}$ & $\begin{array}{c}\text { Gross income } \\
\left(\text { Rs. ha }^{-1}\right)\end{array}$ & $\begin{array}{c}\text { Net income } \\
\left(\text { Rs. ha }^{-1}\right)\end{array}$ & B:C ratio \\
\hline $\mathrm{T}_{1}(\mathrm{CRH}+\mathrm{NP})$ & 22843 & 60300 & 37457 & 2.64 \\
\hline $\mathrm{T}_{2}(\mathrm{BC}+\mathrm{NP})$ & 34093 & 59490 & 25397 & 1.74 \\
\hline $\mathrm{T}_{3}(\mathrm{LN}+\mathrm{NP})$ & 32593 & 54000 & 21407 & 1.66 \\
\hline $\mathrm{T}_{4}(\mathrm{FYM}+\mathrm{NP})$ & 32843 & 65700 & 32857 & 2.00 \\
\hline $\mathrm{T}_{5}(\mathrm{CRH})$ & 20040 & 45000 & 24960 & 2.25 \\
\hline $\mathrm{T}_{6}(\mathrm{BC})$ & 31290 & 46770 & 15480 & 1.49 \\
\hline $\mathrm{T}_{7}(\mathrm{FYM})$ & 30040 & 50430 & 20390 & 1.68 \\
\hline $\mathrm{T}_{8}(\mathrm{LN})$ & 29790 & 44580 & 14790 & 1.50 \\
\hline $\mathrm{T}_{9}(\mathrm{Control})$ & 18790 & 37500 & 18710 & 2.00 \\
\hline $\mathrm{T}_{10}(\mathrm{RDF}-\mathrm{NPK})$ & 21593 & 57000 & 35407 & 2.14 \\
\hline
\end{tabular}

\section{Available soil moisture (Per cent)}

Available soil moisture (per cent) was recorded at a depth of $0-15 \mathrm{~cm}$ is given in Table (6). Among the treatments, enriched charred rice husk treatment recorded highest available soil moisture. It was closely followed by enriched biochar treatment compared to control treatment. The incorporation of charred rice husk and biochar into soil can alter physical properties such as structure, pore size distribution, soil aeration and water holding capacity. In addition, the soil hydrology may be affected by partial or total blockage of soil pores by the smallest particle size fraction of charred rice husk and biochar thereby decreasing water infiltration rates. Hence there was less depletion of soil moisture in the above treatment possibly due to higher moisture retention capacity of enriched biochar. Mapa and Silva (1994) also confirmed the above fact.
Relative water content increased with the application rate of enriched charred rice husk 5 tons $\mathrm{ha}^{-1}$ Table (6) in the experiment. This could be due to the role of charred rice husks as organic fertilizer on better holding the water in the root zone

With respects of the number of nodules was significantly varied among the treatments. The higher number of nodules was recorded in the application of 5 tons ha $^{-1}$ enriched FYM $\left(\mathrm{T}_{4}\right)$ followed by enriched charred rice husk $\left(\mathrm{T}_{1}\right)$ Table (6). The result obtained is similar to Collins and Duke (1981) who revealed that in legumes, potassium deficit may cause inadequate supply of sugars to roots nodules, which greatly reduces rates of $\mathrm{N}_{2}$ fixation and export of bound nitrogen, Seripong (1988) who concluded that phosphorus and rice husk ask improved nodulation in Thailand soils. 


\section{Water uptake by different organic manures application}

The release of water from organic manures is presented in Table (7). The release of water from charred rice husk, biochar, lignite and farm yard manure ceased to exist within 3 days of experiment. The higher water leachate was observed in absolute control treatment, while charred rice husk was lower one. The leachate of water collected on the first day was observed in charred rice husk, biochar, lignite and farm yard manure with 179.33, 185.10, 207.50 and $270.67 \mathrm{ml}$ respectively from the initial addition of $500 \mathrm{ml}$ of water in the column.

On second day, it was observed the lower water release $(179.33 \mathrm{ml}$ from $500 \mathrm{ml})$ from enriched charred rice husk. It was followed by enriched biochar and enriched lignite with 185.10 and $207.50 \mathrm{ml}$ respectively. The same trend was observed in third day also. The control treatment without mixing of organic manures recorded higher release of water $(270.67 \mathrm{ml})$.

The lowest leachate of water that may be due to absorbed water molecule in the microspores of the charred rice husk are held very strongly and released only upon the force exerted by the roots of groundnut which come into contact with organic material. Water held in the organic material utilized by the plants when it is in stress which will help to tide over the drought stress and sustain growing activity of plant. The slow release of water from charred rice husk might be due to enhanced uptake and utilization of more water. Ghanem and Ebaid (2001) reported that increasing irrigation intervals from 4 days consequently water was saved by 8.2 per cent.

\section{Economics of cultivation}

Favorable environment with respect to nutrient and moisture by the addition of enriched organic manures enhanced the productivity of rainfed groundnut resulted higher gross and net income. Among the enriched organic manure treatments, enriched charred rice husk registered maximum net return (Rs. 37457 ha $^{-1}$ ) and $\mathrm{B}: \mathrm{C}$ ratio (2.64) followed by the application of enriched farm yard manure which recorded net return just above variable cost (Rs. 32857 $\mathrm{ha}^{-1}$ ) and B: C ratio (2.00). Which were presented in Table (8).

From the experimental findings it can be concluded that, among the ten treatments, application of enriched farm yard manure at the rate of 5 tons ha $^{-1}$ as basal resulted in higher yield and yield attributes, followed by the enriched bio char and enriched charred rice husk applied at rate of at 5 tons $\mathrm{ha}^{-1}$.

With respect to economics, among the organic manure treatments, the charred rice husk recorded the maximum net returns and income per rupee invested due to the cheaper material cost. Therefore, it would be concluded that application of enriched charred rice husk as a source of potassium at a rate of 5 tons $\mathrm{ha}^{-1}$ is favorable for yield advancements in groundnut. In India charred rice husk is a highly available and cheap manures in large quantities. Hence, application of charred rice husk is best suited to small farming in red soil conditions.

\section{Future line of work}

Optimization of charred rice husk for different agriculture crops should be standardized under different ecosystems.

Long term carbon sequestration potential of charred rice husk in different ecosystem should be studied in detail through long term experiment.

Charred rice husk induced microbial dynamics and its role in nutrient availability mechanism may be studied in detail.

\section{Abbreviations}

CRH-Charred Rice Husk $5 \mathrm{t} \mathrm{ha}^{-1}$, BC- Biochar $5 \mathrm{t} \mathrm{ha}^{-1}$, LN-Lignite $5 \mathrm{t} \mathrm{ha}^{-1}$, FYM- Farmyard Manure $5 \mathrm{t} \mathrm{ha}^{-1}$, N-Nitrogen $10 \mathrm{~kg} \mathrm{ha}^{-1}$, P-Phosphorus $10 \mathrm{~kg} \mathrm{ha}^{-1}$, 
K-Potash $45 \mathrm{~kg}$ ha- ${ }^{1}$,

RDF-Recommended dose of Fertilizer 10:10:45 kg NPK ha ${ }^{-1}$.

\section{References}

Akbari, K.N., G.S. Sutaria, D.S. Hirpara, B.A. Kunjadia and V.N. Patel. 2011. Effect of phosphorus fertilization with and without FYM on groundnut yield and soil fertility under rainfed condition. Legume Res., 25(2):117 -120.

Aliyu, T.H., O.S. Balogun and O.O. Alade. 2011. Assessment of the effect of rate and time of application of rice-husk powder as an organic manures on cowpea (Vigna unguiculata L.) inoculated with cowpea mottle virus. Agric. and Biolo. J. of North America, 2151-7525.

Budhar, M.N. and S.P.Palaniappan. 1994. Effect of incorporation of proceeding cotton on succeeding rice. Indian $\mathrm{J}$. agron., 39(3):461-463.

Budhar,M.N. 2003. Effect of conjunctive and individual application of inorganic fertilizer and organic manure on rainfed ragi. Crop scientists meet. 78 .

Chandrasekaran, R., E. Somasundaram, Mohamed Amanullah, K. Nalini, K. Thirukkumaran and K. Sathyamoorthi. 2007. Response of confectionery groundnut (Arachis hypogaea L.) varieties to farm yard manure. J. of Applied Sci. Res., 3(10):1097-1099.

Dhane, S.S., S.B. Dodaka and S.N. Jadhav. 1996. Evaluation of vermicompost in groundnut production, Maharashtra, India. Int. Arachis Newsletter, 16:52-53.

Directorate of Economics and Statistics. 2009-

10. Department of Agriculture and Corporation, Ministry of Agriculture, Government of India.

Ghanem, S.A. and R.A. Ebaid. 2001. Effect of farm yard manure and water regimes on productivity of rice and succeeding clover crop, Egypt J. Appil. Sci., 16 (16):115128.

Gomez, K.A. and A.A. Gomez. 1984. Statistical Procedures for Agricultural Research. New Delhi, India: John Wiley, 680.

Gupta, A. P., R.S. Patil and Narwal. 1988. Effect of Farmyard manure on Organic carbon, available $\mathrm{N}$ and $\mathrm{P}$ content of soil during different periods of wheat growth. J. Indian Soc. of Soil Sci., 36(2): 269-273.

James, A., L.L. Entry and R.E. Sojka. 2007. Water air and soil pollution. Water air and soil pollut., 180(1): 283- 292.

Kirnak, H., C. Kaya, I. Tas and D. Higgs. 2001. The influence of water deficit on vegetative growth, physiology, fruit yield and quality in eggplants. Bulgarian J. Plant Physio., 7(3-4):4-46.

Mapa, R.B. and A.D.E. Silva. 1994. Effect of organic matter on available water in noncalcic brown soils. Sri lankan J. Agri. Sci., 31:82-92.

Schneider, D., M. Escala, K. Supawittayayothin and N. Tippayawong. 2011. Characterization of biochar from hydrothermal carbonization of bamboo. Internat. J. Energy Environ, 2(4):647-652.

\section{How to cite this article:}

Balasubramanian, P. and Chinnamuthu, C.R. 2017. Effect of Charred Rice Husk on Rainfed Groundnut for Water Retention and Nutrient Management (Arachis hypogaea L.). Int.J.Curr.Microbiol.App.Sci. 6(11): 4123-4133. doi: https://doi.org/10.20546/ijcmas.2017.611.483 